\title{
Primeiros Socorros Psicológicos: Intervenção em crise para eventos de violência urbana
}

\author{
Pedro Goularte Lara ${ }^{1}$ \\ Gustavo Ramos Silva² \\ Luiza Lewgoy Servino ${ }^{3}$ \\ Vítor Corrêa Frimm ${ }^{4}$ \\ Christian Haag Kristensen ${ }^{5}$
}

\section{RESUMO}

Este projeto atende à demanda de estudar alternativas de resposta ao fenômeno prevalente e importante da violência urbana. A partir de estratégias em diferentes níveis - capacitação e intervenção em crise - busca-se esclarecer como melhor auxiliar vítimas de eventos traumáticos. No Rio Grande do Sul, o período de 2014 a 2017 foi marcado por um aumento histórico nos índices de violência, o que colocou a capital Porto Alegre no Ranking das 50 cidades mais violentas do mundo. Nesse período, $22 \%$ dos porto-alegrenses já perderam um familiar por homicídio e a letalidade da polícia cresceu 45\% [1]. Indivíduos em zonas com maior e recorrente exposição à violência urbana estão em risco elevado para o desenvolvimento de psicopatologias incapacitantes. Portanto, é crucial que existam estratégias de intervenção que possam prevenir o desenvolvimento desses transtornos, auxiliando indivíduos nos primeiros momentos após um trauma. Os Primeiros Socorros Psicológicos (PSP) podem ser aplicados por qualquer profissional ou voluntário que tenha sido treinado. Foram desenvolvidos como uma intervenção de apoio psicossocial para prover necessidades básicas e reduzir o estresse inicial causado por eventos traumáticos, de forma a facilitar o processo de recuperação. Assim, eles têm como objetivos principais conectar as pessoas aumentando seu suporte social, promover bem-estar físico e emocional, acalmar e orientar de maneira empática e não-intrusiva, oferecer informações importantes que auxiliem a lidar com os aspectos psicológicos do evento traumático e apoiar respostas adaptativas [2][3]. Trata-se de uma intervenção que se enquadra nas necessidades de contextos com muitas vítimas e poucos recursos de atendimentos disponíveis - como ocorre em muitos contextos brasileiros. Portanto, o presente projeto busca estudar a eficácia de uma intervenção em crise cientificamente informada, os PSP, na redução de sintomas póstraumáticos e de depressão e no aumento da percepção de suporte social. Além disso, profissionais de saúde serão treinados para prover os PSP, e será verificada a eficácia deste treinamento em aumentar seus conhecimentos sobre trauma e intervenção em crise e a sua confiança para realizar PSP. O treinamento ocorrerá periodicamente com os representantes de instituições de saúde. Cada treinamento contará com 10 a 20 profissionais, com duração de 4 horas e incluindo explanação teórica e prática simulada. Além disso, serão coletados dados em intervenções de PSP realizadas com sobreviventes de eventos de violência urbana. Após o momento de intervenção inicial, há o contato por e-mail em 2 ou 3 dias como parte dos PSP, quando será proposta a participação voluntária na pesquisa. Serão aplicados os instrumentos PCL-5, CERQ e ESSS. Será disponibilizado espaço para que o participante comente sobre a influência específica de cada técnica na sua recuperação. Posteriormente, será feito contato por e-mail com os mesmos indivíduos para a realização de follow-ups após 1 e 3 meses. Espera-se que indivíduos que sejam treinados em PSP desempenhem significativamente melhor nos grupos de questões avaliando conhecimentos de trauma e confiança para realizar

\footnotetext{
${ }^{1}$ PUCRS - Pontifícia Universidade Católica do Rio Grande do Sul - Brasil.

2 PUCRS - Pontifícia Universidade Católica do Rio Grande do Sul - Brasil.

3 PUCRS - Pontifícia Universidade Católica do Rio Grande do Sul - Brasil.

4 PUCRS - Pontifícia Universidade Católica do Rio Grande do Sul - Brasil.

5 PUCRS - Pontifícia Universidade Católica do Rio Grande do Sul - Brasil.
} 
intervenção em crise. Quanto aos indivíduos atendidos em PSP, espera-se que apresentem menores chances de desenvolver sintomas e transtornos pós-traumáticos.

Palavras-chave: intervenção em crise, violência urbana, eventos traumáticos

\section{INTRODUÇÃO}

\subsection{Consequências pós-traumáticas da violência urbana}

No Brasil, eventos como homicídios, conflitos armados e assaltos são foco de atenção para políticas públicas de segurança e de saúde de grandes centros urbanos. Isso porque, para além da perda de vidas humanas, a violência causa impacto significativo para a economia nacional [4] e para a saúde das testemunhas e sobreviventes desses eventos [5].

Em um estudo realizado com amostra representativa das cidades de Rio de Janeiro e São Paulo, foi identificado que $90 \%$ dos participantes passaram por ao menos um evento considerado traumático ao longo da vida [5]. Frente a tal prevalência de exposição a traumas, os autores relatam que cerca de $10 \%$ da população desses grandes centros urbanos desenvolveu o Transtorno de Estresse PósTraumático (TEPT) em algum momento de suas vidas. Trata-se de uma reação pós-traumática de intensidade e duração maiores do que o esperado e que provocam sofrimento intenso e prejuízo social e funcional em níveis incapacitantes.

No Rio Grande do Sul, o período de 2014 a 2017 foi marcado por um aumento histórico nos índices de violência, o que colocou a capital Porto Alegre no Ranking das 50 cidades mais violentas do mundo. Nesse período, $22 \%$ dos porto-alegrenses já perdeu um familiar por homicídio e a letalidade da polícia cresceu 45\% [1]. Questiona-se, portanto, como esses índices aumentados de violência se refletem na saúde mental da população de Porto Alegre e região metropolitana.

Indivíduos em zonas com maior e recorrente exposição à violência urbana estão em risco elevado para o desenvolvimento de psicopatologias incapacitantes, como o TEPT [6]. Há achados de que profissionais que trabalham com emergências e resgates têm chance aumentada de desenvolverem reações pós-traumáticas [7]. Conforme o Manual Diagnóstico e Estatístico de Transtornos Mentais (DSM-5), duas reações patológicas altamente incapacitantes são as principais diretamente relacionadas a eventos traumáticos, o Transtorno de Estresse Agudo (TEA) e o TEPT. Seus sintomas incluem memórias involuntárias recorrentes sobre o trauma, alterações negativas no humor e em cognições, evitação de estímulos relacionados à memória do trauma e reatividade acentuada frente a esses estímulos [6]. Além disso, há outras possíveis psicopatologias associadas ao desenvolvimento e curso do TEPT. Dentre as comorbidades mais comuns, encontra-se o Transtorno Depressivo Maior, transtornos de ansiedades variados, e transtornos por uso e dependência de substâncias psicoativas [6].

Dentre os fatores de prevenção e remediação de eventos traumáticos, ressalta-se a importância da percepção de suporte social, cuja ausência é considerada um preditor importante de reações póstraumáticas patológicas [8]. Dada a realidade da violência e dos recursos para intervenção disponíveis nas cidades brasileiras, convém adaptar intervenções eficientes e com potencial de disseminação para que se possa prevenir o desenvolvimento dessas psicopatologias. 
Poucos estudos desenvolvem protocolos eficazes e eficientes de intervenção em situações de crise. Além disso, não foram encontrados estudos brasileiros indicando a existência de respostas institucionais bem estabelecidas e/ou sistematizadas para crises e traumas, não havendo, portanto, um protocolo de intervenção imediata formalizado para profissionais de saúde que atuam em contextos de violência urbana e de vulnerabilidade social. Portanto, o presente projeto busca estudar a eficácia de uma intervenção em crise cientificamente informada, os Primeiros Socorros Psicológicos (PSP), e capacitar profissionais da rede de atenção em saúde de Porto Alegre para proverem este tipo de intervenção adaptada à realidade de violência urbana brasileira. Dessa forma, os PSP surgem como alternativa para reduzir impactos individuais e coletivos por conta da ausência de ferramentas semelhantes na prática cotidiana desses profissionais.

\subsection{Os Primeiros Socorros Psicológicos}

Os PSP são uma estratégia de intervenção que pode ser aplicada por qualquer profissional ou voluntário que tenha sido treinado. Foram desenvolvidos como uma intervenção de apoio psicossocial, para prover necessidades básicas e reduzir o estresse inicial causado por eventos traumáticos de forma a facilitar o processo de recuperação. Até hoje, não foram revisados estudos que sustentem ou refutem empiricamente a eficácia da intervenção em reduzir sintomas pós-traumáticos; entretanto, os elementos e intervenções de PSP são baseados em evidências sólidas sobre fatores de risco e características do processamento de informações após um trauma. Por isso, segundo Dieltjens et al.[9] e Fox et al.[10], embora sua eficácia não seja evidenciada por estudos empíricos, os PSP são recomendados por consenso de experts e são utilizados ao redor do mundo por instituições que prestam auxílio humanitário e em saúde.

A abordagem dos PSPs não presume que os desastres vão causar adoecimento mental em todos os sobreviventes, mas sim que algumas reações iniciais podem aliar-se a estratégias desadaptativas para dificultar a recuperação. Assim, segundo os guias operacionais [2][3], eles têm como objetivos principais conectar as pessoas - aumentando seu suporte social -, promover bem-estar físico e emocional, acalmar e orientar de maneira empática e não-intrusiva, oferecer informações importantes que auxiliem a lidar com os aspectos psicológicos do evento traumático e apoiar respostas adaptativas. Trata-se de uma intervenção que se enquadra nas necessidades de contextos com muitas vítimas e poucos recursos de atendimentos disponíveis - como ocorre em muitos contextos brasileiros.

\subsection{Objetivo e questão problema}

Neste estudo, busca-se estudar alternativas de resposta ao fenômeno prevalente e importante da violência urbana e esclarecer como melhor auxiliar vítimas de eventos traumáticos, atendendo a necessidades básicas e atenuando o sofrimento psíquico. Para tanto, o estudo divide-se estrategicamente em duas frentes: capacitação de profissionais da rede de atenção à saúde de Porto Alegre e região metropolitana; e intervenção direta e coleta de dados de vítimas recentes de violência urbana. Tem como objetivos avaliar a efetividade dos treinamentos quanto ao aprendizado sobre trauma e reações pós-traumáticas, estrutura dos PSP e autoavaliação da capacidade para realizar intervenções em crise; e avaliar o efeito da intervenção de PSP sobre os sintomas pós-traumáticos e de depressão e sobre a percepção de suporte social. 


\section{MÉTODOS E PROCESSOS}

A pesquisa está sendo conduzida por integrantes do grupo de pesquisa Cognição, Emoção e Comportamento, no Núcleo de Estudos e Pesquisa em Trauma e Estresse (NEPTE) da Pontifícia Universidade Católica do Rio Grande do Sul (PUCRS), em colaboração formal com a Secretaria Municipal de Saúde de Porto Alegre (SMS), assim como com colaborações já em andamento com a coordenadoria de residências do Grupo Hospitalar Conceição (GHC) e com a Fundação Municipal de Saúde de Canoas (FMSC). O projeto tem previsão de conclusão em 3 anos.

\subsection{Participantes}

Para a capacitação em Primeiros Socorros Psicológicos, com o apoio da Secretaria Municipal de Saúde de Porto Alegre e da divulgação do NEPTE (Núcleo de Estudos e Pesquisa em Trauma e Estresse), são selecionados profissionais da rede de atenção à saúde da região metropolitana, formando grupos diversificados para realização do treinamento. Espera-se que ao menos 90 profissionais recebam treinamento direto.

Quanto aos indivíduos vítimas de um trauma recente que recebem os PSP, ao fim da intervenção é realizada a combinação de um contato posterior por e-mail em 2 ou 3 dias pelo integrante do grupo de suporte. Nesse contato, os indivíduos atendidos são convidados a participar da pesquisa. São convocados participantes, também, através de folhetos e pôsteres em unidades de saúde e pontos chave de acolhimento de emergência. Diversos profissionais da rede de saúde são solicitados a encaminhar vítimas recentes de trauma ao NEPTE. Além disso, quando há demanda de realização de PSP por parte das instituições de saúde conveniadas, um pesquisador do NEPTE acompanha a equipe de suporte que realizará a intervenção. Após isso, é feito contato por e-mail com os mesmos indivíduos para a realização de follow-ups, para reaplicação dos instrumentos, em 1 e 3 meses. Espera-se a participação de 60 indivíduos nesta parte do estudo.

\subsection{Instrumentos}

Como critério de participação no treinamento em PSP, os profissionais de saúde respondem o "Questionário para avaliação do treinamento de PSP". Trata-se de um questionário, construído na plataforma online 'Qualtrics', desenvolvido para avaliação do aprendizado dos participantes do treinamento adaptado. O questionário é composto por três blocos de questões: o primeiro para avaliar conhecimentos sobre trauma e reações pós-traumáticas (contendo 14 questões), o segundo sobre procedimentos de intervenção em crise e PSP (22 questões) e, por fim, uma autoavaliação sobre aptidão e preparo para realizar intervenções em crise e PSP (13 questões). Esses blocos são precedidos por questões ligadas ao tempo de experiência e tipo de função realizada na área da saúde. Além disso, ao final, o participante é solicitado a descrever sua percepção de autoeficácia em intervenções em crise que já realizou de fato - antes e/ou depois de terem recebido algum tipo de treinamento em intervenção em crise.

Cada participante responde apenas uma parcela das questões em cada bloco, que são escolhidas aleatoriamente, sendo 4 questões no primeiro e terceiro blocos e 8 questões no segundo bloco. Esse método é utilizado para que os questionários tenham uma probabilidade de incluir novas questões em uma reaplicação no mesmo participante. 
O questionário é aplicado nos participantes antes e depois da realização do treinamento, como forma de medir variações no conhecimento geral que os profissionais têm a respeito de eventos traumáticos e intervenções em crise e na autopercepção de capacidade para realizá-las. Será verificado o efeito do treinamento em PSP sobre os três grupos de questões do questionário através de testes de comparação de médias de medidas repetidas. Nos grupos de questões envolvendo conhecimentos (2 primeiros grupos de questões), será utilizado o número de respostas corretas do participante. No terceiro grupo (autoavaliação de aptidão), será calculada uma média de nível de confiança por participante da escala likert utilizada.

No contato realizado com os indivíduos após a intervenção de PSP, é encaminhado o link para acesso à plataforma Qualtrics, com acesso ao termo de consentimento livre e esclarecido (TCLE) e aos instrumentos de avaliação descritos abaixo.

Posttraumatic Stress Disorder Checklist (PCL-5), uma escala que tem o objetivo de mensurar a gravidade dos sintomas pós-traumáticos de acordo com os critérios diagnósticos de TEPT do DSM-5 [11].

Questionário de Regulação Emocional Cognitiva (CERQ), um instrumento de autorrelato para avaliar as estratégias cognitivas de regulação emocional [12].

Escala de Satisfação com o Suporte Social (ESSS), possui a finalidade de avaliar a satisfação com o suporte social recebido, incluindo as dimensões de satisfação com amigos, com a família, relações de intimidade e atividades sociais [13].

Também, são realizadas perguntas a cada participante sobre sua avaliação de como cada grupo de intervenções dos PSP influenciou a apresentação atual de seus sintomas - ou da ausência destes. Além de uma escala likert de 5 pontos (variando de "nem um pouco" a "completamente"), é disponibilizado espaço para que o participante comente sobre a influência específica de cada técnica (i.e., escuta reflexiva, atendimento de necessidades imediatas, estabilização emocional, promoção de segurança, priorização, fortalecimento das redes de apoio social e realização de encaminhamentos) na sua recuperação.

\subsection{Procedimentos e Análise de dados}

Cada treinamento conta com 10 a 20 profissionais, de diferentes áreas de atuação, com a duração de 4 horas e ocorre periodicamente nas dependências da PUCRS. O treinamento, conduzido por integrantes do NEPTE, inclui explanação dos objetivos e procedimentos teóricos dos Primeiros Socorros Psicológicos e práticas simuladas, i. e., exercícios de escuta reflexiva e de manejo de desregulação emocional com role play. Dessa forma, são apresentados desafios possíveis durante a realização de uma intervenção em crise. Além disso, é fornecido material de consulta e uma lista de encaminhamentos para que o conteúdo do treinamento possa ser revisado antes da aplicação da intervenção.

A adaptação realizada para a realidade brasileira sustenta-se em manuais de PSP divulgados por instituições engajadas no manejo de eventos traumáticos e desastres [2][3] e é composta por seis etapas de PSP, definidas pela sigla PREPARA - Preparação, Recepção, Escuta, Priorização, Ação e ReAvaliação [14]. 
A preparação é a etapa em que o provedor deve se preparar e planejar, levar em conta seu nível de conforto com o tipo de trabalho, a sua saúde atual e circunstâncias no trabalho ou na família e estar atento para fornecer autocuidado, respeitando seus limites. Além disso, deve buscar manter uma presença calma e estar atento às diferenças culturais, diversidade e às populações de risco. Na etapa de recepção, o provedor deve se apresentar e perguntar sobre necessidades urgentes como, segurança imediata, ferimentos e ao bem-estar físico, como sono, alimentação e fadiga. Deve-se tomar o cuidado para garantir a confidencialidade e não invadir o espaço pessoal sem autorização.

Na etapa de escuta, é importante validar a experiência subjetiva, ouvir o relato e parafrasear mostrando compreensão e reconhecendo os esforços e acertos do sobrevivente. Em seguida, na priorização, reconhecer quem precisa de cuidado e com maior urgência, identificar sinais de prejuízo funcional e de comportamentos de risco. Devem ser consideradas algumas experiências que podem aumentar a probabilidade de uma resposta disfuncional, como dissociação, risco de suicídio e sentimentos de culpa ou vergonha.

A etapa seguinte, ação, busca estabilizar a excitabilidade psicológica, mitigar o estresse agudo e melhorar a funcionalidade individual, promover segurança e o acesso e fortalecimento da rede de apoio. Por último, na reavaliação, programar um segundo momento de contato para retomar as recomendações e checar se foram utilizadas, além de encaminhar o indicar as possibilidades de continuidade do atendimento - dentro do SUS e com outras instituições.

A realização de intervenções em PSP, por sua natureza, tem um grau elevado de imprevisibilidade em termos de locais e recursos disponíveis. É solicitado pela equipe que realiza a intervenção um espaço físico suficiente para atendimentos individuais e manutenção de sigilo. Além disso, os folhetos educativos são disponibilizados para o grupo de suporte que realiza cada intervenção.

Os integrantes do NEPTE que realizam a intervenção de PSP mantém um registro escrito de particularidades do atendimento, nos moldes de um diário de campo com roteiro estruturado. Nesse registro, são descritas as condições do espaço físico, número de pessoas atendidas e as percepções sobre a efetividade da intervenção e dificuldades na sua execução.

Quanto a análise dos dados, será verificado o efeito do treinamento em PSP sobre os três grupos de questões do questionário de avaliação através de testes de comparação de médias. Nos grupos de questões envolvendo conhecimentos (2 primeiros grupos de questões), será utilizado o número de respostas corretas do participante. No terceiro grupo (autoavaliação de aptidão), será calculada uma média de nível de confiança por participante da escala likert utilizada.

Os questionários preenchidos por indivíduos atendidos em PSP serão comparados, também, através de testes de comparação de médias nos 3 momentos de avaliação. A prevalência de sintomas póstraumáticos e do diagnóstico presumido de TEPT (de acordo com a PCL-5) nos indivíduos atendidos será comparada com índices esperados após eventos traumáticos nessa população. Além disso, as percepções dos prestadores de PSP sobre as características da intervenção serão analisadas em termos de sua frequência e relevância implícita. Assim, será analisada a aproximação da intervenção com sua proposta teórica e serão identificados fatores que influenciem a realização dos PSP. 


\section{RESULTADOS ESPERADOS}

Espera-se que indivíduos que sejam treinados em PSP desempenhem significativamente melhor, de acordo com os instrumentos descritos, nos grupos de questões avaliando conhecimentos de trauma e sofrimento pós-traumático e intervenção em crise, mesmo em avaliações de follow-ups. Além disso, espera-se que expressem mais confiança para a realização de intervenções em crise. Quanto aos indivíduos atendidos em PSP, espera-se que sigam um curso de resiliência (i.e., com redução significativa de sintomas pós-traumáticos em relação às primeiras $48 \mathrm{hs}$ ). Presume-se que a prevalência de TEPT nos follow-ups será menor do que a relatada para populações semelhantes.

\section{DISCUSSÃO}

Atualmente, os Primeiros Socorros Psicológicos são amplamente apoiados em consenso pela opinião de experts, e baseiam-se fortemente em princípios e evidências sobre fatores de risco e características do processamento de informações após um trauma. Entretanto, revisões sistemáticas de literatura apontam que há falta de evidências sólidas que comprovem a eficácia dos PSP, através de estudos empíricos com a população atendida. Isso se dá ao fato de o protocolo só estar alcançando notoriedade recentemente, e devido à dificuldade de reavaliar vítimas do evento após receberem a intervenção. Portanto, este estudo mostra-se desafiador, ao almejar contribuir com evidências empíricas que sustentem ou refutem a eficácia do protocolo de PSP.

Pretende-se, em etapas futuras, incluir neste estudo indivíduos vítimas recentes de eventos traumáticos que não receberam suporte de PSP, como forma produzir um grupo controle. Será possível, assim, desenvolver um parâmetro de comparação com os indivíduos que receberem a intervenção.

\section{REFERÊNCIAS}

[1] Kopittke, "Segurança Pública no Rio Grande do Sul: 2014 a 2017," in Anuario Brasileiro de Segurança Pública: 2014 a 2017. Fórum Brasileiro de Segurança Pública, 2018, pp. 109113. Disponível em: http://www.forumseguranca.org.br/wpcontent/uploads/2018/09/FBSP_ABSP_edicao_especial_estados_faccoes_2018.pdf

[2] M. Brymer, A. Jacobs, C. Layne, R. Pynoos, J. Ruzek, A. Steinberg, ... e P. Watson, Psychological First-Aid: field operations guide, 2nd ed., 2006.

[3] Organização Mundial da Saúde, War Trauma Foundation e Visão Global internacional, Primeiros Cuidados Psicológicos: guia para trabalhadores de campo. OMS: Genebra, 2015.

[4] D. Cerqueira, R. S. de Lima, S. Bueno, D. Coelho, P. P. Alves, M. Reis e F. Merian, Atlas da Violência 2018: Políticas Públicas e Retratos dos Municípios Brasileiros. Rio de Janeiro: Instituto de Pesquisa Econômica Aplicada (IPEA) e Fórum Brasileiro de Segurança Pública (FBSP), 2018.

[5] W. S. Ribeiro, J. D. J. Mari, M. I. Quintana, M. E. Dewey, S. EvansLacko, L. M. P. Vilete, ... e S. B. Andreoli, "The Impact of Epidemic Violence on the Prevalence of Psychiatric 
Disorders in Sao Paulo and Rio de Janeiro, Brazil." PLoS ONE, 2013, vol. 8, n. 5. Disponível em: https://doi.org/10.1371/journal.pone.0063545

[6] American Psychiatric Association, Manual Diagnóstico e Estatístico de Transtornos Mentais - 5a edição. São Paulo: Artmed, 2014.

[7] M. L. B. Almeida, "Prevalência de Estresse Pós-Traumático em Equipes de Resgate: Uma Revisão Sistemática." Psicologia, Saúde \& Doenças, 2012, vol. 13, n. 2, pp. 220-237. Disponível em: http://www.scielo.mec.pt/scielo.php?script=sci arttext\&pid=S164500862012000200007\&lng =pt.

[8] M. J. Woodward, J. Eddinger, A. V. Henschel, T. S. Dodson, H. N. Tran e J. G. Beck, "Social support, posttraumatic cognitions, and PTSD: The influence of family, friends, and a close other in an interpersonal and noninterpersonal trauma group." Journal of Anxiety Disorders, 2015, vol. 35, pp. 60-67. Disponível em: https://doi.org/10.1016/j.janxdis.2015.09.002

[9] T. Dieltjens, I. Moonens, K. Van Praet, E. De Buck e P. Vandekerckhove, "A Systematic Literature Search on Psychological First Aid: Lack of Evidence to Develop Guidelines." PLoS ONE, 2014, vol. 9, n. 12, e114714. Disponível em: https://doi.org/10.1371/journal.pone.0114714

[10] J. H. Fox, F. M. Burkle, J. Bass, F. A. Pia, J. L. Epstein e D. Markenson, "The Effectiveness of Psychological First Aid as a Disaster Intervention Tool: Research Analysis of Peer-Reviewed Literature From 1990-2010." Disaster Medicine and Public Health Preparedness, 2012, vol. 6, n. 3, pp. 247-252. Disponível em: https://doi.org/10.1001/dmp.2012.39

[11] E. de P. Lima, A. G. Vasconcelos, W. Berger, C. H. Kristensen, E. do Nascimento, I. Figueira e M. V. Mendlowicz, "Cross-cultural adaptation of the Posttraumatic Stress Disorder Checklist 5 (PCL-5) and Life Events Checklist 5 (LEC-5) for the Brazilian context." Trends in Psychiatry and Psychotherapy, 2016, vol. 38, n. 4, pp. 207-215. Disponível em: https://doi.org/10.1590/2237-6089-2015-0074

[12] J. L. Schäfer, B. R. Cibils Filho, T. C. de Moura, V. C. Tavares, A. X. Arteche e C. H. Kristensen, "Psychometric properties of the Brazilian version of the Cognitive Emotion Regulation Questionnaire." Trends in Psychiatry and Psychotherapy, 2018, vol. 40, n. 2, pp. 160-169. https://doi.org/10.1590/2237-6089-2017-0074

[13] M. N. Baptista, A. S. Baptista e E. C. Torres, "Associação entre suporte social, depressão e ansiedade em gestantes." Revista de Psicologia Da Vetor Editora, 2006, vol. 7, n. 1, pp. 39-48.

[14] G. S. Everly, O. Lee McCabe, N. L. Semon, C. B. Thompson e J. M. Links, "The Development of a Model of Psychological First Aid for Non-Mental Health Trained Public Health Personnel." Journal of Public Health Management and Practice, 2014, vol. 20, pp. S24-S29. Disponível em: https://doi.org/10.1097/PHH.0000000000000065 